\title{
Parabolic Hall effect due to copropagating surface modes
}

\author{
M. Breitkreiz* \\ Dahlem Center for Complex Quantum Systems and Fachbereich Physik, Freie Universität Berlin, 14195 Berlin, Germany
}

(Received 21 November 2019; accepted 4 March 2020; published 19 March 2020)

\begin{abstract}
Real-space separations of countermoving states to opposite surfaces or edges are associated with different types of Hall effects, such as the quantum, spin, or the anomalous Hall effect. Some systems provide the possibility to separate a fraction of countermovers in a completely different fashion: surface states propagating all in the same direction, balanced by countermoving bulk states, realized, e.g., in Weyl metals with intrinsically or extrinsically broken inversion and time-reversal symmetries. In this Rapid Communication we show that these copropagating surface modes are associated with a specific Hall effect-a parabolic potential profile in the direction perpendicular to and in its magnitude linear in the applied field. While in two-dimensional (2D) systems the parabolic potential profile is directly measurable, in 3D the resulting voltage between the bulk and surface is measurable in the geometry of a hollow cylinder. Moreover, the parabolic Hall effect leads to characteristic signatures in the longitudinal conductivity.
\end{abstract}

DOI: 10.1103/PhysRevResearch.2.012071

Introduction. The condensed-matter realization of Weyl fermions [1-9] has attracted very much interest in the past years, due in large part to the realization of chiral Landau levels, moving parallel or antiparallel to the magnetic field, depending on the Weyl-fermion chirality [10]. In a crystal, the two chiralities appear pairwise, separated in momentum space, which complicates an identification of Weyl-specific transport phenomena such as the chiral magnetic effect [11-14].

Tendentiously it is more promising when chiral states are separated not (or not only) in momentum but in real space-a situation well known from the separation of countermovers to opposite surfaces in topological insulators [15]. Here, the favorable situation associated with the real-space separation is the clear signature in the form of a quantum (spin) Hall effect $[16,17]$. A noteworthy equivalent in the field of Weyl metals is the anomalous Hall effect (AHE) [18,19]—a voltage drop in the direction perpendicular to both the direction of the current flow and the intrinsic magnetization, in the absence of an external magnetic field [20-22]. The mechanism of the AHE in Weyl metals can indeed be understood in terms of chiral surface states-a pairwise connection of Weyl Fermi surfaces of opposite chirality by two Fermi arcs [23], localized at opposite surfaces and moving in opposite directions, which intuitively explains the AHE as the contribution of Fermi arcs in the case of a potential difference between the surfaces [8,24] (see Fig. 1). Remarkably, the presence of a finite density of diffusive bulk states does not obscure the surface-state driven AHE.

\footnotetext{
*breitkr@physik.fu-berlin.de

Published by the American Physical Society under the terms of the Creative Commons Attribution 4.0 International license. Further distribution of this work must maintain attribution to the author(s) and the published article's title, journal citation, and DOI.
}

In this Rapid Communication we focus on a different and much less explored separation of countermovers in real space: forward movers homogeneously distributed in the bulk and back movers localized at the surface. This can be realized in two-dimensional (2D) systems [25] (edge states have been called "antichiral" in this case), including transition metal dichalcogenide monolayers [25], exciton-polariton systems [26], and in 3D Weyl metals [27,28]. Comparing to the well-studied case of counterpropagating surface modes, the question arises whether copropagating surface modes can also be associated with a characteristic Hall effect.

We show that the answer is positive-copropagating surface modes give rise to a Hall response that is characterized by a quadratic spatial dependence of the chemical potential

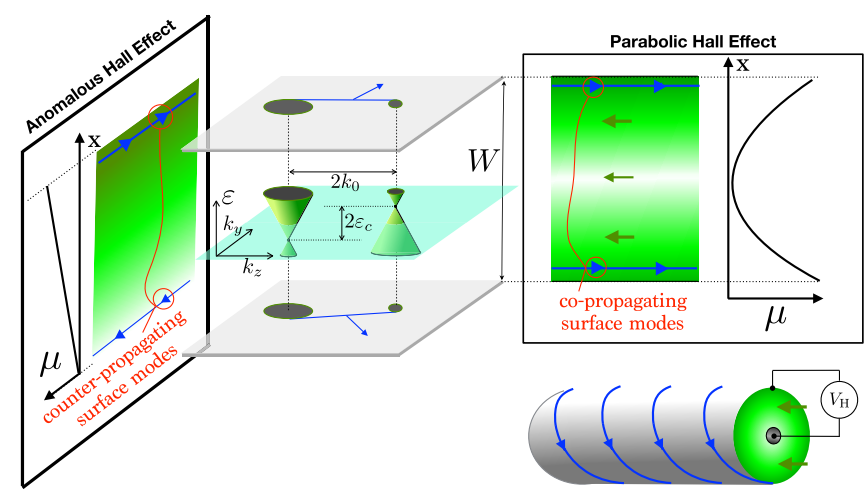

FIG. 1. Weyl-metal slab with two Weyl cones separated in energy and in momentum, connected by Fermi arcs at the surfaces, illustrated in a mixed momentum/real space. An anomalous Hall effect-a linear drop of the chemical potential $\mu$ between the surfaces-occurs if current is led in the plane of counterpropagating surface modes. The parabolic Hall effect occurs when current is led in the plane of copropagating surface modes, leading to a quadratic spatial dependence of $\mu$, measurable in the geometry of a hollow cylinder between the inner and outer surfaces. 
$\mu$, transverse to the applied field. The general mechanism can be understood as follows. In the bulk, the current density of chiral charge carriers (the fraction of bulk charges that in equilibrium compensate the current of copropagating surface states) changes proportionally to the local chemical potential, $\delta \boldsymbol{j}_{c} \propto \delta \mu$. Together with the coexisting diffusive bulk charges flowing according to the diffusion equation $j_{n} \propto \nabla \mu$, the total current must be divergence free in the steady state, hence $\nabla^{2} \mu \propto-\hat{j_{c}} \cdot \nabla \mu$, where $\hat{j_{c}}$ is the direction of the chiral bulk current. According to this relation, if a homogeneous electric field $\boldsymbol{E}=-\nabla \mu$ is applied along $\hat{\boldsymbol{j}}_{c}$, the chemical potential assumes a quadratic spatial dependence in the direction perpendicular to the applied field. Physically, the parabolic Hall field ensures a charge conserving redistribution of charge, which is pumped by the applied field at the surface and in the bulk in opposite directions. This is what we call the parabolic Hall effect (PHE). Note that this effect is still linear in the driving field and thus distinct from effects called the "nonlinear Hall effect" [29].

In the following, we explore the effect in detail. We focus on a minimal 3D model of a Weyl metal (Fig. 1), which, while allowing a direct conclusion on the simpler case of $2 \mathrm{D}$, requires additional calculations to show how the resulting Hall voltage can be measured in 3D systems.

Model. Measuring energy in units of $\hbar v$, where $v$ is the Fermi velocity, and length in units of the lattice constant, the Hamiltonian we consider reads

$$
H=-\sigma_{x} i \partial_{x}+k_{y} \sigma_{y}+m\left(k_{z}\right) \sigma_{z}+\varepsilon_{c} \eta\left(k_{z}\right),
$$

where $\sigma_{i}$ are spin Pauli matrices, $m\left(k_{z}\right)=\left(k_{z}^{2}-k_{0}^{2}\right) / 2 k_{0}$, and $\eta\left(k_{z}\right)=\tanh \left(2 k_{z} / k_{0}\right)$, featuring two Weyl nodes with chirality \pm at momentum $k_{x, y}=0, k_{z}= \pm k_{0}$, and energy $\varepsilon \approx \pm \varepsilon_{c}$.

We focus on the case of two well-separated Weyl cones with vanishing corrections to the linear dispersion and a constant velocity $v$ at the Fermi level $\varepsilon_{F}$, hence $\varepsilon_{c},\left|\varepsilon_{F}\right| \ll k_{0}$. The explicit form of $m\left(k_{z}\right)$ and $\eta\left(k_{z}\right)$ is unimportant as long as these requirements are fulfilled.

Considering a slab of width $W$, the quantum numbers are $\boldsymbol{\kappa}=\left(q, k_{y}, k_{z}\right)$, where $q$ is the solution of

$$
\frac{m\left(k_{z}\right)}{q} \tan (W q)+1=0
$$

coming from the boundary condition of the slab [30]. Throughout this work we assume $W \gg 1$, in which case the solution for $q$ can be divided into three groups: (i) the group of bulk states, given by the quasicontinuous set $q=$ $(n+0.5) \pi / W, n=0,1,2, \ldots$, (ii) surface states with the imaginary (and hence exponentially decaying) solution $q=$ $i m\left(k_{z}\right)$ for $m\left(k_{z}\right)<0$, and (iii) chiral bulk states with the solution $q=0$ for $m\left(k_{z}\right)>0$. The dispersion reads

$$
\varepsilon_{\kappa}=\varepsilon_{c} \eta\left(k_{z}\right) \pm \sqrt{q^{2}+k_{y}^{2}+\left[m\left(k_{z}\right)\right]^{2}}
$$

and the equienergy contours are illustrated in Fig. 2. Note that surface states (ii) and chiral bulk states (iii) merge at $m\left(k_{z}\right)=$ 0 , building a single closed contour.

Since the $x$ dependence of the wave functions is given by $\exp (i q x)$, the finite penetration depth of surface states is $1 / \operatorname{Im} q$. The velocity of a wave packet at state $\boldsymbol{\kappa}$ can thus be written as $\boldsymbol{v}_{\boldsymbol{\kappa}}=\operatorname{Re}\left[\left(\partial_{q}, \partial_{k_{y}}, \partial_{k_{z}}\right) \varepsilon_{\boldsymbol{\kappa}}\right]$ and the free spectral

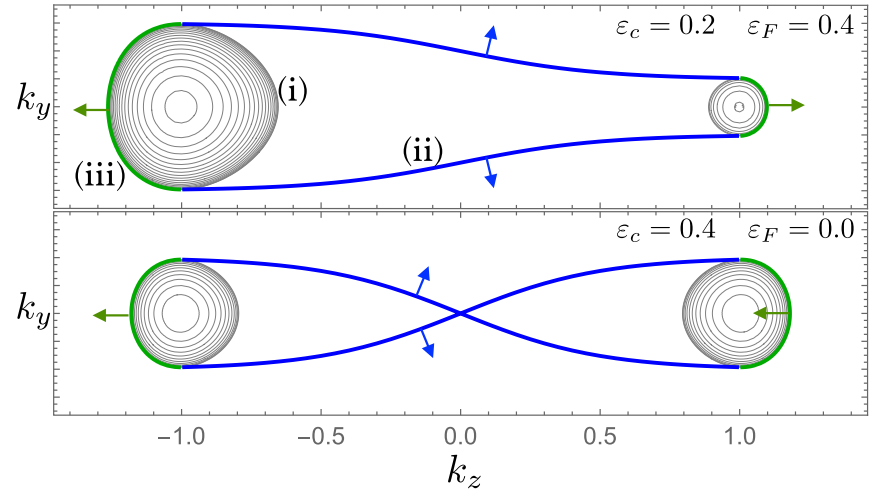

FIG. 2. Fermi-level states for the Weyl metal of width $W=100$, $k_{0}=1$, and two different combinations of $\varepsilon_{F}$ and $\varepsilon_{c}$. The gray, blue, and green states correspond to (i) the normal bulk state, (ii) surface states, and (iii) chiral bulk states, respectively. Velocity directions are indicated by arrows.

function in the center-of-mass coordinate $x$, and the $W \gg 1$ limit reads

$$
\begin{gathered}
A(\boldsymbol{\kappa}, x, \omega)=2 \pi \delta\left(\omega-\varepsilon_{\boldsymbol{\kappa}}\right) \rho(\boldsymbol{\kappa}, x), \\
\rho(\boldsymbol{\kappa}, x)= \begin{cases}1, & \operatorname{Im} q=0, \\
2 W m\left(k_{z}\right) e^{ \pm 2 m\left(k_{z}\right)(x \mp W / 2)}, & \operatorname{Im} q \neq 0,\end{cases}
\end{gathered}
$$

where $\operatorname{Im} q=0$ and $\operatorname{Im} q \neq 0$ distinguish bulk and surface states, and \pm corresponds to surface states at $x= \pm W / 2$. Despite the divergence of penetration at $m\left(k_{z}\right)=0$, the surfacestate spectral weight averaged over all states at the surface is strongly localized, the characteristic length scale being $1 / k_{0} \sim 1$. Since all other length scales will be considered to be much larger, we approximate the $\operatorname{Im} q \neq 0$ case in (5) as $\rho(\kappa, x) \approx W \delta(x \mp W / 2)$ in the following.

The density of bulk states at the Fermi level of the cone \pm and the total bulk density $n_{n}=\sum_{ \pm} n_{n \pm}$ read, respectively,

$$
n_{n \pm}=\frac{\left(\varepsilon_{F} \mp \varepsilon_{c}\right)^{2}}{\pi v h}, \quad n_{n}=2 \frac{\varepsilon_{F}^{2}+\varepsilon_{c}^{2}}{\pi v h} .
$$

We also define the density of chiral bulk states and the 2D density of surface states of a single surface,

$$
n_{c \pm}=\frac{\left|\varepsilon_{F} \mp \varepsilon_{c}\right|}{2 v h W}, \quad n_{s}=\frac{k_{0}}{\pi v h},
$$

respectively. In accord with $W \gg 1$, we assume that $n_{n}$ is much larger than $n_{c \pm}$ and $n_{s} / W$.

Parabolic Hall effect. To explore the transport behavior in linear response, we aim to find a solution for a statedependent deviation of the chemical potential from the Fermi energy $\mu(\boldsymbol{\kappa})$ with an arbitrary spatial profile along the $x$ direction, given the boundary condition of a homogeneous force field applied in the $z$ direction, $\partial_{z} \mu(\kappa)=E$. Furthermore, we assume elastic scattering from a weak disorder potential. To focus on qualitative features, we take the scattering amplitudes to be different only between Fermi-level states of the different types $i \in[n+, c+, s+, n-, c-, s-]$. Using the quantum Boltzmann formalism [31] and employing the standard semiclassical approximation scheme (see Supplemental 
Material for details [32]), we obtain

$$
\begin{aligned}
\nabla \cdot\left(\boldsymbol{j}_{n \pm}+\boldsymbol{j}_{c \pm}\right)= & \pm \gamma_{n-n+}\left(\mu_{n-}-\mu_{n+}\right) \\
& +\gamma_{s n \pm} s(x)\left(\mu_{s}-\mu_{n \pm}\right), \\
s(x) \nabla \cdot j_{s \pm}= & s(x)\left[\gamma_{s n+}\left(\mu_{n+}-\mu_{s \pm}\right)\right. \\
& \left.+\gamma_{s n-}\left(\mu_{n-}-\mu_{s \pm}\right)\right], \\
j_{n \pm}= & -n_{n \pm} D \nabla \mu_{n \pm},
\end{aligned}
$$

where $\gamma_{i j}=\Gamma_{i j} n_{i} n_{j}$ and $\Gamma_{i j}$ is the scattering rate, $\mu_{i}=$ $\langle\mu(\kappa)\rangle_{i}$ is the local chemical potential averaged over the Fermi-level states $i, \boldsymbol{j}_{i}=n_{i}\left\langle\boldsymbol{v}_{\boldsymbol{\kappa}} \mu(\boldsymbol{\kappa})\right\rangle_{i}$ is the nonequilibrium current-density contribution of the states $i, D$ is the bulk diffusion constant, and $s(x)=\sum_{+} \delta(x \pm W / 2)$.

To linear order in $E$ and using translation invariance in the $y$ direction, the divergence of the chiral-bulk and the surface particle currents simplify to

$$
\nabla \cdot \boldsymbol{j}_{c \pm}= \pm \frac{\varepsilon_{F} \mp \varepsilon_{c}}{\pi h W} E, \quad \nabla \cdot \boldsymbol{j}_{s \pm}=\frac{\varepsilon_{c}}{\pi h} E .
$$

We rewrite Eqs. (8) by considering the differential Eqs. (8a) away from the boundary $[s(x)=0]$, together with $(8 \mathrm{c})$ and (9),

$$
\pm \frac{\varepsilon_{F} \mp \varepsilon_{c}}{\pi h W} E-n_{n \pm} D \partial_{x}^{2} \mu_{n \pm}= \pm \gamma_{n+n-}\left(\mu_{n-}-\mu_{n+}\right) .
$$

This is supplemented with boundary conditions, given by integration of (8) over an infinitesimal distance at both boundaries and assuming vanishing current through the boundary,

$$
\begin{aligned}
j_{n+}^{x}( \pm W / 2)= & \mp \gamma_{s n+}\left[\mu_{s \pm}-\mu_{n+}( \pm W / 2)\right], \\
j_{n-}^{x}( \pm W / 2)= & \mp \gamma_{s n-}\left[\mu_{s \pm}-\mu_{n-}( \pm W / 2)\right], \\
\frac{\varepsilon_{c}}{\pi h} E= & \gamma_{s n+}\left[\mu_{n+}( \pm W / 2)-\mu_{s \pm}\right] \\
& +\gamma_{s n-}\left[\mu_{n-}( \pm W / 2)-\mu_{s \pm}\right] .
\end{aligned}
$$

Assuming that an external contact would couple to all bulk states with equal probability it would probe the averaged chemical potential

$$
\mu_{n}=\frac{n_{n+} \mu_{n+}+n_{n-} \mu_{n-}}{n_{n}},
$$

for which Eqs. (10) and (11) readily provide the solution

$$
\mu_{n}=-\frac{n_{s} v}{n_{n} D} \frac{\varepsilon_{c}}{k_{0}} \frac{x^{2}}{W} E+z E .
$$

Sticking to the interpretation that $E$ is an applied field in the $z$ direction, the response lies in the first term in (13), which exhibits the PHE - a quadratic spatial dependence on the transverse coordinate $x$ and a linear dependence on the magnitude of the applied field $E$. The roles of applied and induced fields are of course interchangeable so that a finite magnitude of the transverse potential would induce a finite longitudinal field $E$ and with that a longitudinal current (which will be calculated below).

Measuring the Hall voltage. In contrast to the ordinary Hall effect where the potential varies linearly and is fully characterized by a voltage between opposite surfaces, the PHE voltage occurs between one surface and the bulk and varies quadratically with the distance. While in a $2 \mathrm{D}$ system the

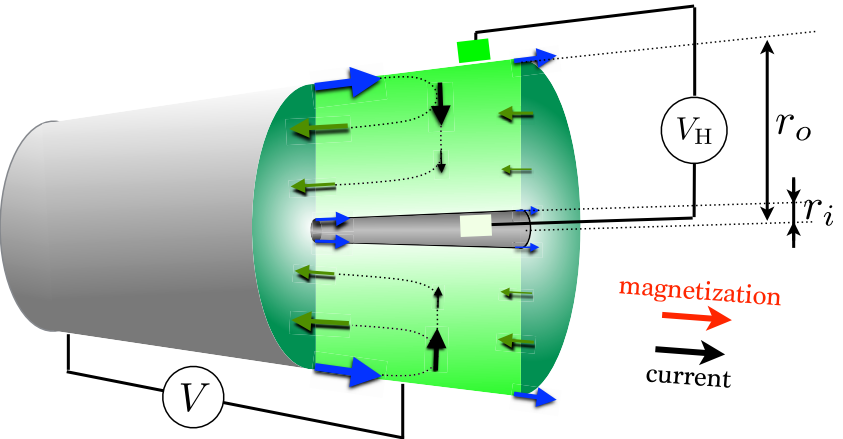

FIG. 3. Weyl metal in the geometry of a hollow cylinder with a small inner radius. When current is led along the cylinder and the magnetization, the PHE voltage is induced between the inner and the outer surface. Arrows indicate local current flows of chiral bulk (green), surface (blue), and diffusive bulk (black) particles.

parabolic voltage profile can be measured directly, in a 3D system a contact inside the sample necessarily introduces an inner surface so that the actual geometry for such a measurement is (in its most simple realization) that of a hollow cylinder (see Fig. 3) with an inner radius $r_{i}$ and an outer radius $r_{o}$. A straightforward modification of the above formalism [32] [essentially consisting in the replacements $W \rightarrow r_{o}-r_{i}$, $\left.x \rightarrow r, \partial_{x} j_{n}^{x} \rightarrow\left(\partial_{r}+1 / r\right) j_{n}^{r}\right]$ leads to the solution

$$
\mu_{n}(r)=-\frac{n_{s} v}{n_{n} D} \frac{\varepsilon_{c}}{k_{0}}\left(\frac{r^{2} / 2}{r_{o}-r_{i}}-\frac{r_{o} r_{i}}{r_{o}-r_{i}} \ln \frac{r}{r_{i}}\right) E+z E .
$$

We quantify the voltage between the inner and the outer surfaces via the resulting Hall angle $\theta_{\mathrm{PHE}} \equiv\left[\mu_{n}\left(r_{o}\right)-\right.$ $\left.\mu_{n}\left(r_{i}\right)\right] /\left(r_{o}-r_{i}\right) E$. In the limit $r_{o} \gg r_{i}$ we obtain

$$
\theta_{\mathrm{PHE}}=-\frac{n_{s} v}{n_{n} D} \frac{\varepsilon_{c}}{2 k_{0}} \text {. }
$$

Note that the first factor, $n_{s} v / n_{n} D \equiv \theta$, is the Hall angle of the AHE $[19,24]$, which is thus related to $\theta_{\mathrm{PHE}}$ by the ratio of energy versus momentum separation of the Weyl nodes (energy in units of $\hbar v$ ).

Conductivity. We first note that general symmetry considerations $[33,34]$ fix the form of the infinite-system conductivity tensor of our system to

$$
\sigma=\left(\begin{array}{ccc}
\sigma^{x x} & \sigma^{x y} & 0 \\
-\sigma^{x y} & \sigma^{x x} & 0 \\
0 & 0 & \sigma^{z z}
\end{array}\right)
$$

which follows from rotation symmetry around $z$ and symmetry with respect to time reversal combined with $C_{2}$ rotation around an $x-y$ plane axis, corresponding to the Laue group $\infty 2^{\prime}$ [33]. As discussed above, the model exhibits AHE in the $x-y$ plane, with a Hall angle $\sigma^{x y} / \sigma^{x x}=\theta$, related to the intrinsic magnetization in the $z$ direction. The PHE is instead found in the plane parallel to $z$ but it evidently does not manifest itself in a finite $\sigma^{x z}$ or $\sigma^{y z}$, which is in agreement with our result that the potential difference in the $x$ direction between outer surfaces vanishes.

In the following we show that the PHE still manifests itself in the infinite-system conductivity-it gives rise to an anomalous term in $\sigma^{z z}$, which is size dependent but finite in 
the infinite-system limit. The current contribution of normal bulk states in response to the field $E$ is obtained from $(8 \mathrm{c})$ as $j_{n}^{z}=-n_{n} D E$. This would be the only contribution to $\sigma^{z z}$ that one would obtain for an infinite system based on the Drude formula; we denote it as $\sigma_{0}^{z z} \equiv j_{n}^{z} / E$. The additional contributions of chiral bulk states and surface states, neglecting corrections of order $1 / W$, can be written as $j_{c \pm}^{z}= \pm\left(\varepsilon_{F} \mp\right.$ $\left.\varepsilon_{c}\right) \mu_{n \pm} / \pi h W$ and $j_{s \pm}^{z}=\delta(x \mp W / 2) \mu_{s \pm} \varepsilon_{c} / \pi h$, respectively. Inserting $\mu_{i}$ as solutions of (10) and (11), the full conductivity is given by $\sigma^{z z}=\left(j_{n}^{z}+\bar{j}_{c}^{z}+\bar{j}_{s}^{z}\right) / E$, where the bar denotes averaging over $x$. In terms of $\sigma_{0}^{z z}$ we obtain

$$
\begin{aligned}
& \frac{\sigma^{z z}}{\sigma_{0}^{z z}}=1+\frac{4}{3} \theta_{\mathrm{PHE}}^{2}\left(1+\frac{6 l_{n s}}{W \theta}\right)\left(1+\frac{\varepsilon_{F}^{2}}{\varepsilon_{c}^{2}} \xi\right), \\
& \xi=\frac{\frac{2 l_{c}}{W}-\frac{\left(\frac{2 l_{c}}{W}\right)^{2}}{\frac{l_{n s}}{\theta l_{c}}+\operatorname{coth} \frac{W}{2 l_{c}}}}{\frac{W}{6 l_{c}}+\frac{l_{n s}}{\theta l_{c}}}= \begin{cases}0, & l_{c} \ll W, \\
1, & l_{c} \gg W, l_{n s} / \theta,\end{cases}
\end{aligned}
$$

where $l_{n s}=v / \Gamma_{s n} n_{n}$ is the relaxation length of surface states and $l_{c}=\sqrt{D / \Gamma_{n+n-} n_{n}}$ is the internode relaxation length [35]. In the infinite-system limit we obtain $\sigma^{z z}=$ $\left(1+4 \theta_{\mathrm{PHE}}^{2} / 3\right) \sigma_{0}^{z z}$, demonstrating a remarkable deviation from the Drude behavior. We expect that the same correction can be derived based purely on the bulk Hamiltonian, e.g., using Kubo formalism. We leave this for future work, noting that similarly exchangeable derivations from an infinite- and a finite-system perspective have been demonstrated for the AHE in Refs. [19,24].

The finite-size correction in the first brackets is similar to the finite-size correction of the AHE in Ref. [24] and is due to the vanishing dissipation of surface states if their scattering length becomes large compared to the width $W$. The second brackets correspond to a finite-size correction which occurs if the system is not electron-hole compensated $\left(\varepsilon_{F} \neq 0\right)$, in which case the applied field induces an occupation imbalance between the Weyl nodes leading to a prolonged relaxation time. The resistivity decrease saturates even if $l_{c} \rightarrow \infty$ because internode relaxation also happens indirectly via surface states.

Discussion. Our calculations have shown that copropagating surface modes and the related counterpropagating bulk states (which we here call chiral) give rise to a parabolic transverse potential profile. We made the realistic assumption that the density of coexisting normal bulk states (spatially not separated countermovers) is finite and hence $\sim W$ times larger than the number of surface states, where $W$ is the width in units of the lattice constant. Nevertheless, the spatial separation of surface and chiral bulk states by $\sim W$ compensates this so that the PHE survives the limit $W \rightarrow \infty$. Besides the Hall voltage, we have identified an anomalous term in the longitudinal conductivity, which can be interpreted as the precursor of the anomalously large conductance in the quantum regime of localized normal bulk states [25,36].

We exemplified the parabolic Hall effect on a model for a Weyl metal with intrinsically broken inversion and timereversal symmetries, which shows a homogeneous chiral charge density in the bulk [37]. Several methods have been proposed to induce (or change) the chiral charge density in Weyl metals externally. For example, internode charge pumping via parallel electric and magnetic fields can effectively shift the energies of the Weyl nodes as

$$
\varepsilon_{c} \rightarrow \varepsilon_{c}+\frac{e^{2}}{h^{2}} \frac{\tau}{n_{n}} E B,
$$

where $\tau$ is the internode relaxation time, which is assumed to be much larger than intranode relaxation [11]. Another interesting possibility to induce and control the PHE is the application of a strain-induced pseudomagnetic field [28,36,38], which allows one to change the density of chiral states even without an electric field and independent of the internode relaxation.

Furthermore, the derived unconventional dependence of the conductivity on the system size and the scattering amplitudes as a consequence of the PHE is an interesting starting and reference point for investigations of the conductivity with regard to temperature dependence in the case of phononmediated scattering [39], or the scaling behavior with the system size [40]. It is worth noting that in a time-reversal invariant Weyl metal, the mechanism of a reduced and sizedependent resistivity due to a doubling of the AHE [24] also applies to the PHE. The PHE voltage would vanish but the associated suppression of the resistivity would remain when the coupling between the time-reversed states is sufficiently weak. The suppression would set in when the width $W$ becomes comparable to the characteristic scattering length quantifying the coupling of time-reversed states.

Acknowledgments. The author would like to thank Piet W. Brouwer, Tobias Meng, and Rodrigo G. Pereira for valuable discussions. This research was supported by Grant No. 18688556 of the Deutsche Forschungsgemeinschaft (DFG, German Research Foundation).
[1] S.-Y. Xu, N. Alidoust, I. Belopolski, Z. Yuan, G. Bian, T.-R. Chang, H. Zheng, V. N. Strocov, D. S. Sanchez, G. Chang, C. Zhang, D. Mou, Y. Wu, L. Huang, C.-C. Lee, S.-M. Huang, B. Wang, A. Bansil, H.-T. Jeng, T. Neupert et al., Science 349, 613 (2015).

[2] S.-Y. Xu, N. Alidoust, I. Belopolski, Z. Yuan, G. Bian, T.-R. Chang, H. Zheng, V. N. Strocov, D. S. Sanchez, G. Chang, C. Zhang, D. Mou, Y. Wu, L. Huang, C.-C. Lee, S.-M. Huang, B. Wang, A. Bansil, H.-T. Jeng, T. Neupert et al., Nat. Phys. 11, 748 (2015).
[3] B. Q. Lv, H. M. Weng, B. B. Fu, X. P. Wang, H. Miao, J. Ma, P. Richard, X. C. Huang, L. X. Zhao, G. F. Chen, Z. Fang, X. Dai, T. Qian, and H. Ding, Phys. Rev. X 5, 031013 (2015).

[4] S. Borisenko, Q. Gibson, D. Evtushinsky, V. Zabolotnyy, B. Büchner, and R. J. Cava, Phys. Rev. Lett. 113, 027603 (2014).

[5] M. Neupane, S.-Y. Xu, R. Sankar, N. Alidoust, G. Bian, C. Liu, I. Belopolski, T.-R. Chang, H.-T. Jeng, H. Lin, A. Bansil, F. Chou, and M. Z. Hasan, Nat. Commun. 5, 3786 (2014). 
[6] Z. K. Liu, B. Zhou, Y. Zhang, Z. J. Wang, H. M. Weng, D. Prabhakaran, S. K. Mo, Z. X. Shen, Z. Fang, X. Dai, Z. Hussain, and Y. L. Chen, Science 343, 864 (2014).

[7] J. Xiong, S. K. Kushwaha, T. Liang, J. W. Krizan, M. Hirschberger, W. Wang, R. J. Cava, and N. P. Ong, Science 350, 413 (2015).

[8] N. P. Armitage, E. J. Mele, and A. Vishwanath, Rev. Mod. Phys. 90, 015001 (2018).

[9] B. Yan and C. Felser, Annu. Rev. Condens. Matter Phys. 8, 337 (2017).

[10] H. B. Nielsen and M. Ninomiya, Phys. Lett. B 130, 389 (1983).

[11] A. A. Burkov, Annu. Rev. Condens. Matter Phys. 9, 359 (2018).

[12] A. A. Burkov, Phys. Rev. B 96, 041110(R) (2017).

[13] S. Nandy, G. Sharma, A. Taraphder, and S. Tewari, Phys. Rev. Lett. 119, 176804 (2017).

[14] R. D. dos Reis, M. O. Ajeesh, N. Kumar, F. Arnold, C. Shekhar, M. Naumann, M. Schmidt, M. Nicklas, and E. Hassinger, New J. Phys. 18, 085006 (2016)

[15] X. L. Qi and S. C. Zhang, Rev. Mod. Phys. 83, 1057 (2011).

[16] K. von Klitzing, Rev. Mod. Phys. 58, 519 (1986).

[17] M. König, S. Wiedmann, C. Brüne, A. Roth, H. Buhmann, L. W. Molenkamp, X.-L. Qi, and S.-C. Zhang, Science 318, 766 (2007).

[18] A. A. Burkov and L. Balents, Phys. Rev. Lett. 107, 127205 (2011).

[19] A. A. Burkov, Phys. Rev. Lett. 113, 187202 (2014).

[20] T. Suzuki, R. Chisnell, A. Devarakonda, Y.-T. Liu, W. Feng, D. Xiao, J. W. Lynn, and J. G. Checkelsky, Nat. Phys. 12, 1119 (2016).

[21] E. Liu, Y. Sun, L. Müchler, A. Sun, L. Jiao, J. Kroder, V. Süß, H. Borrmann, W. Wang, W. Schnelle, S. Wirth, S. T. B. Goennenwein, and C. Felser, Nat. Phys. 14, 1125 (2018).

[22] P. Li, J. Koo, W. Ning, J. Li, L. Miao, L. Min, Y. Zhu, Y. Wang, N. Alem, C.-X. Liu, Z. Mao, and B. Yan, arXiv:1910.10378.

[23] L. Balents, Physics 4, 36 (2011).

[24] M. Breitkreiz and P. W. Brouwer, Phys. Rev. Lett. 123, 066804 (2019).
[25] E. Colomés and M. Franz, Phys. Rev. Lett. 120, 086603 (2018).

[26] S. Mandal, R. Ge, and T. C. H. Liew, Phys. Rev. B 99, 115423 (2019).

[27] P. Baireuther, J. A. Hutasoit, J. Tworzydło, and C. W. J. Beenakker, New J. Phys. 18, 045009 (2016).

[28] D. I. Pikulin, A. Chen, and M. Franz, Phys. Rev. X 6, 041021 (2016).

[29] I. Sodemann and L. Fu, Phys. Rev. Lett. 115, 216806 (2015).

[30] N. Bovenzi, M. Breitkreiz, T. E. O’Brien, J. Tworzydło, and C. W. J. Beenakker, New J. Phys. 20, 023023 (2018).

[31] G. D. Mahan, Many-Particle Physics (Kluwer Academic, New York, 2000).

[32] See Supplemental Material at http://link.aps.org/supplemental/ 10.1103/PhysRevResearch.2.012071 for details of the derivation and solution of transport equations for the slab and the hollow-cylinder geometry.

[33] W. H. Kleiner, Phys. Rev. 142, 318 (1966).

[34] M. Seemann, D. Ködderitzsch, S. Wimmer, and H. Ebert, Phys. Rev. B 92, 155138 (2015).

[35] S. A. Parameswaran, T. Grover, D. A. Abanin, D. A. Pesin, and A. Vishwanath, Phys. Rev. X 4, 031035 (2014).

[36] J. Behrends, R. Ilan, and J. H. Bardarson, Phys. Rev. Research 1, 032028 (2019).

[37] More generally, a nonlinear potential is produced by chiral states acting as particle sources or drains when a driving field $E_{\|}$ is aligned with their motion, $\nabla^{2} \mu=E_{\|} / \lambda$, where the characteristic length $\lambda$ given by the relative densities and mobilities of the chiral and diffusive particles might be spatially inhomogeneous.

[38] A. G. Grushin, J. W. F. Venderbos, A. Vishwanath, and R. Ilan, Phys. Rev. X 6, 041046 (2016).

[39] R. G. Pereira, F. Buccheri, A. De Martino, and R. Egger, Phys. Rev. B 100, 035106 (2019).

[40] C. Zhang, Z. Ni, J. Zhang, X. Yuan, Y. Liu, Y. Zou, Z. Liao, Y. Du, A. Narayan, H. Zhang, T. Gu, X. Zhu, L. Pi, S. Sanvito, X. Han, J. Zou, Y. Shi, X. Wan, S. Y. Savrasov, and F. Xiu, Nat. Mater. 18, 482 (2019). 